\title{
Computational Neurogenetic Modeling: A Methodology to Study Gene Interactions Underlying Neural Oscillations
}

\author{
Lubica Benuskova, Simei Gomes Wysoski, and Nikola Kasabov, Senior Member, IEEE
}

\begin{abstract}
We present new results from Computational Neurogenetic Modeling to aid discoveries of complex gene interactions underlying oscillations in neural systems. Interactions of genes in neurons affect the dynamics of the whole neural network model through neuronal parameters, which change their values as a function of gene expression. Through optimization of the gene interaction network, initial gene/protein expression values and neuronal parameters, particular target states of the neural network operation can be achieved, and statistics about gene interaction matrix can be extracted. In such a way it is possible to model the role of genes and their interactions in different brain states and conditions. Experiments with human EEG data are presented as an illustration of this methodology and also, as a source for the discovery of unknown interactions between genes in relation to their impact on brain activity.
\end{abstract}

\section{INTRODUCTION}

$\mathrm{G}$ enetic studies show that human EEG has a strong genetic basis [1-3]. That is, the EEG spectral characteristics are strongly preserved within families as well as in individuals. In other words, spectral characteristics of brain electrical oscillations are highly conserved in one individual but vary between individuals even when recorded under the same conditions. Moreover, genetically related individuals have similar features in electrical activity of their brains [1]. In the presented work, we want to use our new method of Computational Neurogenetic Modeling (CNGM) to model the dependency of neural electrical activity upon internal gene dynamics in order to account for the spectral differences in the EEG in different brain conditions and different individuals. The goal of the paper is to present how to apply a hierarchical model of EEG generation all the way up from genes to local field potential generation to reveal genetic differences in normal and abnormal EEG. Our hierarchical model of EEG generation introduced in $[4,5]$ has the following components:

- neuronal genes and their expression levels;

- protein expression levels;

- interactions between genes;

- receptor and ion channels related functions;

- generation of electric signals;

This work was supported by the NERF grant X0201 funded by FRST, New Zealand and by KEDRI funds (www.kedri.info).

L.Benuskova (phone: 0064-9-9219343, fax: 0064-9-9219501; e-mail: lbenusko@aut.ac.nz), S.G. Wysoski and N. Kasabov are all with the Knowledge Engineering and Research Discovery Research Institute (KEDRI), Auckland University of Technology, Auckland, New Zealand (email: \{swysoski, nkasabov\}@ aut.ac.nz).
- generation of a local field potential (LFP).

We will introduce step by step: (1) how to link parameters of a neural model to activities of genes/proteins, (2) which genes/proteins are to be included in the model, (3) how to model gene/protein dynamics, (4) how to validate the model on the real brain data, (5) what kind of new and useful information about neural gene interactions can be derived by means of CNGM.

\section{AN ABSTRACT CNGM}

\section{A. An Abstract Gene Regulatory Network Model}

Let $\mathbf{G}=\left\{G_{l}, G_{2}, \ldots, G_{n}\right\}$ be the set of genes in a Gene Regulatory Network (GRN) (Fig. 1). We assume that the expression level of each gene $g_{j}(t+\Delta t)$ is a nonlinear function of expression levels of all the genes in $\mathbf{G}$, so that

$$
g_{j}(t+\Delta t)=\sigma\left(\sum_{k=1}^{n} w_{j k} g_{k}(t)\right)
$$

This nonlinear equation is inspired by discrete models from $[6,7]$, based on the fact that gene expression data are gathered in discrete time intervals [8].

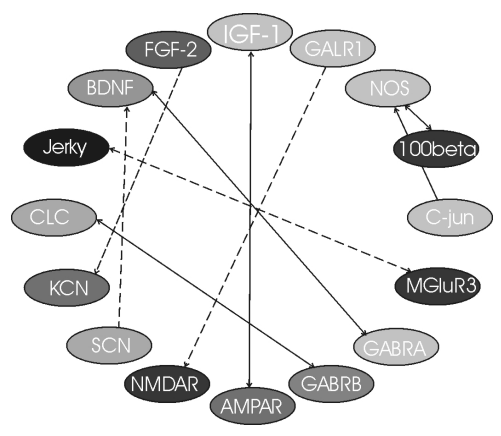

Fig. 1. An abstract model of gene regulatory network. Solid (dashed) lines denote positive (negative) interactions between genes, respectively. Level of grey reflects the level of gene expression at a given time instant. The lines mean a logical connection, rather than a chemical, a physical or physiological one.

We work with normalized gene expression values, expressed by a nonlinear sigmoid $\sigma(\mathrm{x}) \in(0,1)$. The coefficients $w_{i j} \in$ $(-5,5)$ are elements of the square matrix $\mathbf{W}=\left\{w_{i j}\right\}$ of gene interaction weights. The latter borders have been chosen experimentally in order to lead to various types of nonlinear dynamics, i.e. constant as well as periodic, quasi-periodic and chaotic. It is important to identify what the sign of an interaction means, i.e. whether $w_{i j}>0$ or $w_{i j}<0$. The positive interaction means that upregulation (downregulation) of one gene supports the upregulation 
(downregulation) of the other gene, respectively. The negative interaction means that upregulation (downregulation) of one gene leads to the downregulation (upregulation) of the other gene, respectively. Initial values of gene expressions are small random values, i.e. $g_{j}(0) \in(0$, $0.1)$. In the current model we assume a simple scenario where: (1) one protein is coded by one gene; (2) relationship between protein levels and gene expression levels are linear; (3) protein levels lie between the minimal and maximal values. Thus, the protein level $p_{j}(t+\Delta t)$ is expressed at an abstract level by:

$$
p_{j}(t+\Delta t)=\left(p_{j}^{\max }-p_{j}^{\min }\right) \sigma\left(\sum_{k=1}^{n} w_{j k} g_{k}(t)\right)+p_{j}^{\min }
$$

The delay $\Delta t$ corresponds to the regular interval when both gene and protein expression values would be measured experimentally. It is a common practice to derive $\mathbf{W}$ based on all gene expression data being collected at the same time intervals $\Delta t[9]$.

Since neuronal parameters like excitation and inhibition are mediated by the action of neurotransmitters through the corresponding receptor proteins in the postsynaptic membrane, we will link the values of these parameters to the levels of proteins that mediate them. Thus in our model, some protein levels will be directly related to neuronal parameters $P_{j}$ such that

$$
P_{j}(t)=P_{j}(0) p_{j}(t)
$$

Where $P_{j}(0)$ is the initial value of the neuronal parameter at time $t=0$ taken from the value range listed in Table II and $p_{j}(t)$ is the level of the corresponding protein at time $t$. In such a way the gene/protein dynamics is linked to the dynamics of ANN. The abstract CNGM model from formulas (1)-(3) is a general one and can be integrated with any other neural network model, depending on what kind of neural activity one wants to model. In the presented model we have made several simplifying assumptions:

- Each neuron has the same gene regulatory network $(\mathrm{GRN})$, i.e. the same genes and the same interaction gene matrix $\mathbf{W}$.

- Each GRN starts from the same initial values of gene expressions.

- There is no feedback from neuronal activity or any other external factors to gene expression levels or protein levels.

All neuronal parameters and their correspondence to particular proteins in the model presented in this paper are summarized in Table I. They are selected based on their relevance to the spiking activity of a neuron [10-12]. Several parameters like the amplitude and time constants of one postsynaptic potential (PSP) are linked to one protein level however their initial values for Eq. (3) are different. Values of firing threshold parameters $P_{\vartheta}$ depend equally on the levels of three ion channels, such that

$$
P_{\vartheta}(t)=P_{\vartheta}(0)\left(\frac{1 / p_{N a C}(t)+p_{K C}(t)+p_{C l C}(t)}{3}\right)
$$

TABLE I

Neuronal Parameters AND Their Related PROteIns

\begin{tabular}{ll}
\hline \hline \multicolumn{1}{c}{ Neuronal parameter } & \multicolumn{1}{c}{ Protein* } \\
AMPLITUDE AND TIME CONSTANTS OF & \\
\hline Fast excitation PSP & AMPAR \\
Slow excitation PSP & NMDAR \\
Fast inhibition PSP & GABRA \\
Slow inhibition PSP & GABRB \\
Firing threshold & SCN, KCN, CLC \\
\hline
\end{tabular}

*Abbreviations: PSP $=$ postsynaptic potential, AMPAR $=($ aminomethylisoxazole- propionic acid $)$ AMPA receptor, NMDAR $=(\mathrm{N}-$ methyl-D-aspartate acid) NMDA receptor, GABRA = (gammaaminobutyric acid) $\mathrm{GABA}_{\mathrm{A}}$ receptor, $\mathrm{GABRB}=\mathrm{GABA}_{\mathrm{B}}$ receptor, $\mathrm{SCN}=$ Sodium voltage-gated channel, $\mathrm{KCN}=$ kalium (potassium) voltage-gated channel, CLC $=$ chloride channel.

Moreover, besides the genes coding for the proteins listed in Table I, we include in our GRN nine more genes that are not directly linked to neuronal information-processing parameters, but are reported to be related to some neuronal activities [8, 13-18]. These genes are: c-jun, mGLuR3, Jerky, BDNF, FGF-2, IGF-I, GALR1, NOS, S100beta. We have included them for future studies. For now, they only represent the generality of our approach.

\section{B. Spiking Neural Networks (SNN) for CNGM}

The spiking neural network (SNN) used in our CNGM has been first described in $[4,5]$. The model neural network has two layers (Fig. 2a).

(a)
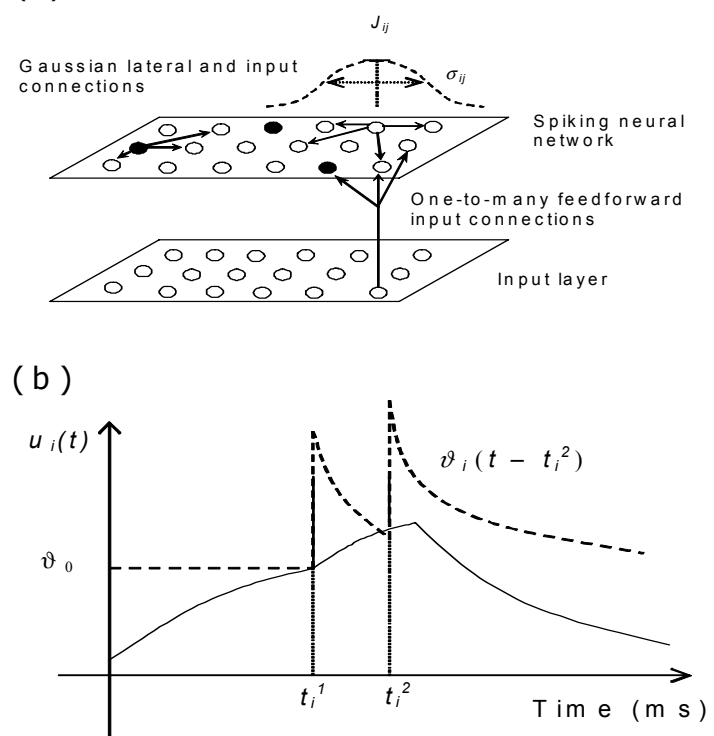

Fig. 2. (a) SNN is comprised of two layers, representing the thalamus (input layer) and cerebral cortex (output SNN). About $10-20 \%$ of $n=$ 120 neurons are inhibitory neurons that are randomly positioned on the grid (filled circles). (b) Spiking neuron model. When the membrane potential $u_{i}(t)$ of the $i^{\text {th }}$ spiking neuron reaches the firing threshold $\vartheta_{i}(t)$ at time $t_{i}^{k}$, the neuron fires an output spike. $\vartheta_{i}(t)$ rises after each output spike and decays back to the resting value $\vartheta_{0}$.

The input layer represents the thalamus (the main subcortical sensory input relay to cortex) and the output layer represents cerebral cortex. We simulate the awake resting state of this system. Individual model neuron is based 
upon a classical Spike Response Model (SRM) [19]. In SRM, the state of a neuron $i$ is described by the state variable $u_{i}(t)$ that can be interpreted as a total somatic PSP. When $u_{i}(t)$ reaches the firing threshold $\vartheta_{i}(t)$ from below, neuron $i$ fires, i.e. emits a spike (see Fig. 2b). The moment of $v_{i}(t)$ crossing defines the firing time $t_{i}$ of an output spike. The value of the state variable $u_{i}(t)$ is the weighted sum of all PSPs $=\varepsilon_{i j}$ :

$$
u_{i}(t)=\sum_{j \in \Gamma_{i} t_{j} \in F_{j}} J_{i j} \varepsilon_{i j}\left(t-t_{j}-\Delta_{i j}^{a x}\right)
$$

Where $\Gamma_{i}$ is the pool of neurons presynaptic to neuron $i$, and $F_{\mathrm{i}}$ is the set of times $t_{j}<t$ when presynaptic spikes occurred. $\Delta^{\mathrm{ax}}$ is an axonal delay between neurons $i$ and $j$ which increases with Euclidean distance between neurons. The weight of synaptic connection from neuron $j$ to neuron $i$ is denoted by $J_{i j}$. It takes positive (negative) values for excitatory (inhibitory) connections, respectively. Lateral and input connections have weights that decrease in value with distance from neuron $i$ according to a Gaussian formula while the connections themselves can be established at random (with probability $p=0.5$ ). The positive kernel $\varepsilon_{i j}$ expresses a particular type of PSP evoked on neuron $i$ when a presynaptic neuron $j$ from the pool $\Gamma_{i}$ fires at time $t_{j}$, such that

$$
\mathcal{E}_{i j}^{t \text { tipe }}(s)=A^{\text {synapse }}\left(\exp \left(-\frac{s}{\tau_{\text {decay }}^{t \text { tpe }}}\right)-\exp \left(-\frac{s}{\tau_{\text {rise }}^{t \text { tipe }}}\right)\right)
$$

where $\tau_{\text {decay }}$ and $\tau_{\text {rise }}$ are time constants of the decay and rise of the double exponential, respectively, $A$ is the amplitude, and type denotes one of the following: fast excitation, fast_inhibition, slow_excitation, and slow_inhibition. These types of PSPs are based on neurobiological data [11]. Thus, in each excitatory and inhibitory synapse, there is a fast and slow component of PSP, based on particular types of postsynaptic receptors listed in Table I. Immediately after firing the output spike at $t_{i}$, neuron's firing threshold $\vartheta_{i}(t)$ increases $k$-times and then returns to its initial value $\vartheta_{0}$ in an exponential fashion:

$$
\vartheta_{i}\left(t-t_{i}\right)=k \times \vartheta_{0} \exp \left(-\frac{t-t_{i}}{\tau_{\text {decay }}^{\vartheta}}\right)
$$

Where $\tau_{\text {decay }}$ is the time constant of the threshold decay. In such a way, absolute and relative refractory periods are modelled. Firing threshold parameters that are threshold resting value, decay time constant and magnitude of its rise after spike generation, $k$, depend on levels of three ion channels for sodium, kalium and chloride (Table I) [20, 21]. External inputs have only fast excitatory components weighted by input weights, such that

$$
u_{i}^{\text {ext }} \text { input }^{\text {in }}(t)=J_{i}^{\text {ext }}{ }_{\text {input }} \varepsilon_{i}(t)
$$

External inputs from the input layer are added to the right hand side of Eq. 4 at each time step. We employed series of random input spikes generated in the input layer neurons with an average frequency between $10-20 \mathrm{~Hz}$, thus simulating the asynchronous thalamic activity in the awake resting state of the brain.

\section{Simulation AND ANALYsis of SNN OutPut}

EEG is the sum of many LFPs mainly from the cerebral cortex [22]. In our SNN, we define LFP as an average of all instantaneous membrane potentials of neurons in the SNN output layer, i.e. $\Phi(\mathrm{t})=(1 / \mathrm{N}) \Sigma u_{i}(t)$. In order to perform a fair comparison we evaluated both the LFP of the SNN and the EEG signal by means of the fast Fourier method (FFT) [23]. However, should a better description of time and frequency be needed, wavelet transformation can be used instead. We base this comparison of SNN LFP and EEG on evidence that brain LFPs have the same spectral characteristics as EEG [24]. Our target signal is the resting human EEG. Spectral characteristics of the resting awake EEG are the same during the day. Thus any point of daily gene dynamics should lead to the electrical signal with the same spectral characteristics. Therefore we will compress the daily gene dynamics into a time scale of the EEG measurement interval that is $\mathrm{T}=1 \mathrm{~min}$ in order to find the desired gene interaction matrix $\mathbf{W}$. As a result of the compression of gene dynamics into the time interval of EEG measurement, we set up the interval of gene and protein changes $\Delta t$ in Eq. (1) and (2) to $1 \mathrm{~s}$. The time step of updating of neural network dynamics will be $1 \mathrm{~ms}$. The FFT of the LFP/EEG signal is done for clinically relevant frequency bands. These bands are: delta $(0.5-3.5 \mathrm{~Hz})$, theta (3.5-7.5 Hz), alpha $(7.5-12.5 \mathrm{~Hz})$, beta $1(12.5-18 \mathrm{~Hz})$, beta $2(18-30 \mathrm{~Hz})$, gamma $(>30 \mathrm{~Hz})$. Based on FFT we calculate the relative intensity ratios (RIR) for the above frequency bands. RIR for the $k^{\text {th }}$ frequency band is defined as

$$
R I R_{k}=\frac{I_{k}}{I}
$$

Where $I_{k}$ is the power of the $k^{\text {th }}$ frequency band in the signal and $I$ is the total power of the signal. RIRs change over time and we calculate the vector of average values to obtain our objective function for $\mathbf{W}$ optimization (see Fig. 3).

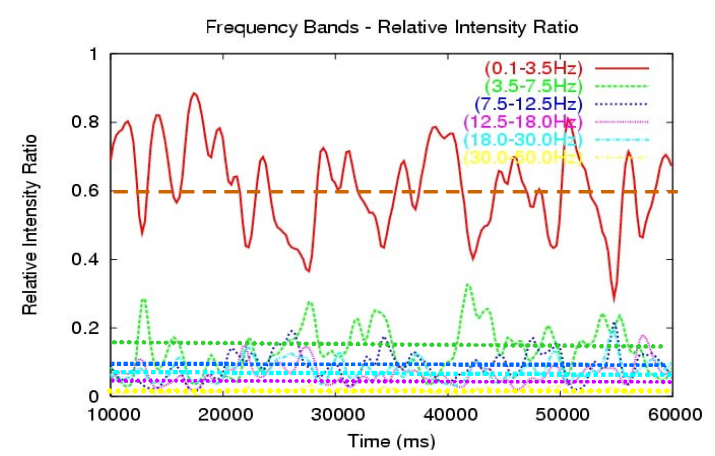

Fig. 3. RIRs for clinically relevant EEG frequency bands change over time. Average values over the measurement interval (dashed lines) constitute desired values to be achieved by the simulated evolution of the gene interaction matrices, W. Sampling rate was $256 \mathrm{~Hz}, \mathrm{Min} / \mathrm{Max}$ frequency $=0.1 / 50 \mathrm{~Hz}$, respectively. 


\section{OptimizATION OF GRN}

We want to achieve the desired SNN output through optimization of the model 294 parameters. We are optimizing the interaction matrix $\mathbf{W}$ between 16 genes of GRN (Fig. 1), initial values of neural parameters and gene expressions, architectural parameters of SNN (except the total number of neurons, spike delays and probability of establishing a synaptic connection) and input frequency to the SNN. All model parameters and the value ranges for initial values $P_{j}(0)$ in Eq. (3) to choose from are listed in Table II. The value ranges were selected based on neurobiological studies and models [10-12] and were further adjusted by experimentation so that the resulting SNN activity was neither too low (no spikes) or too high (too many spikes). In order to find an optimal GRN so that the frequency characteristics of the LFP of the associated SNN are similar to the brain EEG characteristics, we use the following simple optimization procedure:

1. Generate a new CNGM with randomly generated values of coefficients for the GRN matrix $\mathbf{W}$, initial gene expression values $\mathbf{g}(0)$, initial values of parameters $\mathbf{P}(0)$, and other model parameters;

2. Run $\mathrm{CNGM}$ over a period of time $\mathrm{T}=1 \mathrm{~min}$ and record the LFP for the associated SNN;

3. Calculate the average spectral characteristics, i.e. the average RIR vector of the LFP using FFT;

4. Compare the RIR vector of LFP to the average RIR vector of the target EEG signal. Evaluate the closeness of the LFP signal to the target EEG signal characteristics by means of Euclidean distance between the actual and desired RIR vector.

5. Store the CNGM model if it matches the EEG spectral characteristics, let us say, if the Euclidean distance between the RIR vectors is smaller than 0.1 .

6. Repeat the procedure until a sufficient number of solutions is found for statistical analysis.

7. Analyse the GRN interaction matrices $\mathbf{W}$ for statistically significant gene interactions that lead to the desired behavior.

Our objective function in this optimization procedure is based on the average vector of relative intensity ratios (RIRs) for the defined clinically relevant frequency bands. We calculate the average RIRs over the relevant period of measurement (i.e., $\mathrm{T}=1 \mathrm{~min}$ in this case) and use this vector of values find optimal parameters of our model. Thus, any EEG or LFP signal can be characterized by vector of five numbers expressing the average RIRs of particular frequency bands over some time interval. During our optimization we try to find such gene networks that will lead to the SNN LFP with average RIR vector as closest as possible to the average RIR vector of the target human EEG in terms of Euclidean distance. We have expected as was really the case, that in the end of optimization several CNGMs fit the target EEG data. This is not surprising as in the brain the EEG consists of numerous summated sources. Thus, there may be many possible source mixing schemes of
TABLE II

MODEL PARAMETERS AND THEIR VALUE RANGES

\begin{tabular}{lc}
\hline \hline \multicolumn{1}{c}{ Model parameter } & Value range \\
\hline Fast excitation: Amplitude & $0.5-3.0$ \\
Rise / decay time constant (ms) & $5 / 5-10$ \\
Slow excitation: Amplitude & $0.5-4.0$ \\
rise / decay time constants (ms) & $10-20 / 30-50$ \\
Fast inhibition: Amplitude & $4-8$ \\
rise / decay time constants (ms) & $5-10 / 20-30$ \\
Slow inhibition: Amplitude & $5-10$ \\
rise / decay time constants (ms) & $-80 / 50-150$ \\
Resting firing threshold, & $19-25$ \\
decay time constant (ms) / rise k-times & $5-50 / 2-5$ \\
Proportion of inhibitory neurons & $0.15-0.2$ \\
Probability of external input firing & $0.011-0.019$ \\
Peak/sigma of external input weight & $5-10 / 0.1-2$ \\
Peak/sigma of lateral exc weights & $5-14 / 2-8$ \\
Peak/sigma of lateral inh weights & $10-60 / 4-10$ \\
Unit delay in e/i spike propagation & $1 \mathrm{~ms} / 2 \mathrm{~ms}$ \\
Probability of connection & 0.5 \\
Number of neurons & 120 \\
Interaction weights between genes & $(-5,+5)$ \\
Initial expression of any gene & $(0.0,0.1)$ \\
Normalized expression of any gene & $(0.0,1.0)$ \\
\hline \hline
\end{tabular}

underlying LFPs. This would also produce a similar result for the FFT. In our model however, each LFP is related to a different GRN, therefore we need to find those gene interactions which are crucial for the reproduction of LFP with desired spectral characteristics. We will analyse resulting gene interaction matrices Ws for statistically significant gene interactions as it is described on actual results in the next section.

\section{KNOWLEDGE EXTRACTION FROM CNGM}

During optimization we have generated $\mathrm{N}=770 \mathrm{CNGM}$ solutions with random gene interaction matrices $\mathbf{W s}$, random initial gene expressions and other values of parameters randomly generated from allowed intervals of values (see e.g. Table II). Since more than one optimal solution of CNGM leads to the SNN LFP similar to the desired target EEG signal, we are interested in distribution or statistics of gene interactions between genes in the GRN interaction matrix W. In particular, we would like to discover whether interactions between pairs of genes are positive or negative, that is whether upregulation of one gene expression leads to the upregulation of the other gene expression or to the downregulation of its expression, respectively. We visualize the frequency of positive and negative gene interactions in all relevant solutions of CNGM as levels of grey in each element of a summary matrix $\mathbf{W}$ of gene interactions. When the frequency of positive interactions between two genes equals the frequency of negative interactions in all interaction matrices, the cell is filled with grey. The more often we have a positive (negative) interaction among all the solutions the darker (lighter) the cell will be, respectively. Distribution of positive versus negative gene interactions between pairs of genes for all $\mathrm{N}=770$ randomly generated solution is depicted in Fig. 4a. We can see that the distribution of positive and negative gene interactions in all 
770 random solutions is almost uniform therefore all cells are uniformly grey.

(a)

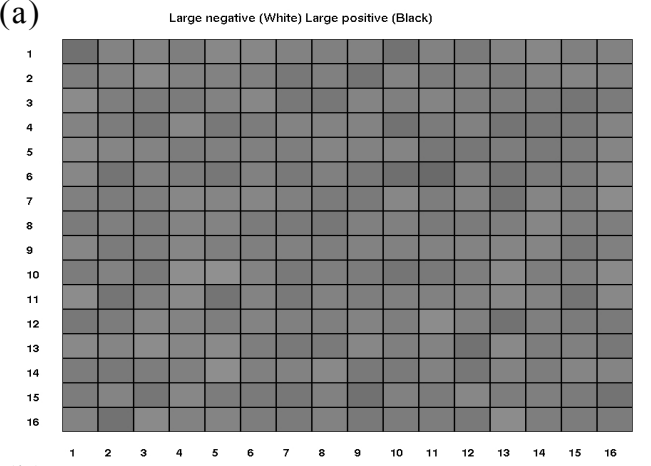

(b)

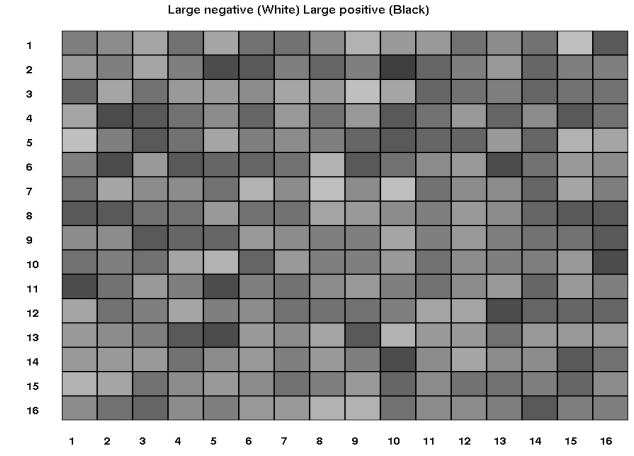

(c )

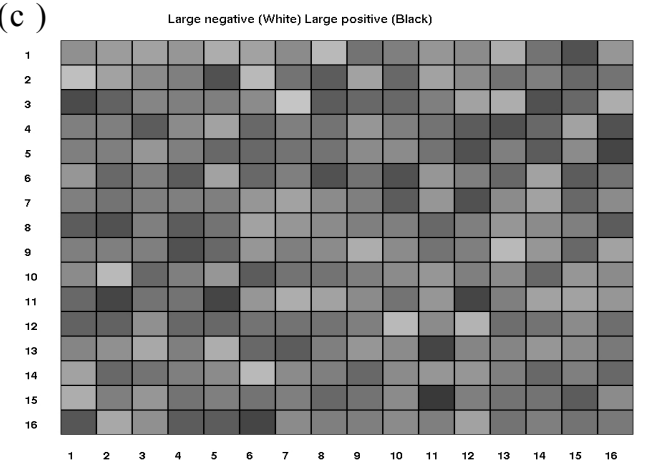

Fig. 4. Levels of grey in each element of a summary matrix $\mathbf{W}$ of gene interactions reflect the frequency of positive and negative gene interactions, respectively, in all relevant solutions leading to the LFP with spectral characteristics similar to the target EEG. Numbers denote the genes in GRN. The bigger is the frequency of positive (negative) interactions, the darker (lighter) is the cell, respectively. (a) Frequencies of positive and negative interactions in 770 randomly generated interaction matrices $\mathrm{W}$ are almost equal. (b) Summary $\mathbf{W}$ of LFPs similar to the normal resting EEG. (c) Summary $\mathbf{W}$ of LFPs similar to 2-3 Hz large wave EEG in CAE seizure. Legend: $1=\mathrm{c}$-jun, $2=$ mGluR3, $3=$ GABRA, $4=$ GABRB, $5=$ AMPAR, $6=$ NMDAR, $7=\mathrm{SCN}, 8=\mathrm{KCN}, 9=\mathrm{CLC}, 10=$ jerky, $11=\mathrm{BDNF}, 12=\mathrm{FGF}-2$, $13=$ IGF-1, $14=$ GALR1, $15=$ NOS, $16=$ S100beta.

We have performed the optimization procedure of CNGM for two kinds of EEG signal. The first target EEG was the resting EEG from a normal healthy adult kindly supplied by Dr. Ian Kirk from University of Auckland, New Zealand. The target vector of average RIR values for objective
TABLE III

STATISTICALLY SIGNIFICANT GENE INTERACTIONS

\begin{tabular}{lccc}
\hline \hline $\begin{array}{l}\text { LFP similar } \\
\text { to the target } \\
\text { EEG for }\end{array}$ & $\begin{array}{c}\text { Significantly } \\
\text { POSITIVE gene } \\
\text { interactions } \\
w(j \rightarrow k)\end{array}$ & $\begin{array}{c}\text { Significantly } \\
\text { NEGATIVE gene } \\
\text { interactions } \\
w(j \rightarrow k)\end{array}$ & $\begin{array}{c}\text { Level } \\
\text { of } X^{2} \\
\text { stat. } \\
\text { signific } \\
\text { ance } \alpha\end{array}$ \\
\hline Normal & & SCN $\rightarrow$ NMDAR & 0.05 \\
resting & none & SCN $\rightarrow$ KCN & 0.05 \\
EEG & & GABRA $\rightarrow$ CLC & 0.05 \\
& & NMDAR $\rightarrow$ KCN & 0.05 \\
2-3 Hz & & & \\
large-wave & & GABRA $\rightarrow$ SCN & 0.025 \\
EEG as in & none & & \\
seizure & &
\end{tabular}

function was: $\operatorname{RIR}_{1}(\delta, \theta, \alpha, \beta 1, \beta 2, \gamma)=(0.6,0.14,0.09,0.07$, $0.08,0.02$ ) (see e.g. Fig. 3). Among 770 random solutions, 20 Ws matrices led to an SNN LFP with spectral characteristics very close to the target EEG signal in terms of Euclidean distance between the RIR vectors being smaller than 0.1. The distribution of positive versus negative gene interactions is illustrated in Fig. 4b. The second target EEG was an artificial $2-3 \mathrm{~Hz}$ large wave signal resembling the Slow Wave Discharge (SWD) EEG observed in the Childhood Absence Epilepsy (CAE) $[25,26]$. There were 22 CNGM solutions of $\mathbf{W}$ that led to such an LFP signal. Statistics of positive versus negative gene interactions from these 22 solutions is illustrated in Fig. 4c.

To discover the knowledge, e.g. to find out what these optimized solutions for a particular condition have in common, we have calculated how many times the interactions between genes become positive and how many times the gene interactions become negative in $20 \mathrm{Ws}$ leading to LFP similar to normal EEG, and in 22 Ws leading to LFP similar to $2-3 \mathrm{~Hz}$ large-amplitude EEG in CAE. We have used the basic frequency statistical analysis, namely the $X^{2}$-statistic, to estimate which gene interactions were statistically significantly positive and negative. Table III summarizes statistically significant gene interactions of a particular sign for those genes, which are directly related to information-processing neural parameters.

\section{VALidation of CNGM Results}

How we can prove that the knowledge extracted from CNGM is correct? Direct test would be to gather gene/protein data and extract the underlying GRN interactions by means of reverse engineering methods. In our particular case study of human EEG, this would not be possible due to ethical reasons. However, we could perform the same study on animal data and compare the predictions from CNGM with the gene interactions discovered by other methods, like for instance reverse engineering. In this section we will present an indirect partial validation of predicted gene interactions by means of neurobiological knowledge. In Ws leading to LFP resembling the normal EEG, there are several statistically significant negative gene interactions. First of all, the interaction between GABRA towards CLC is significantly negative in $\mathbf{W s}$ producing LFP 
similar to normal EEG. GABRA is the inhibitory receptor associated with ion channel for chloride. CLC is the ion channel for chloride that participates in maintaining the ionic and voltage balance by letting $\mathrm{Cl}^{-}$ions into the cell. To function properly, a neuron must establish a dynamic balance between excitation and inhibition so that it can respond to its inputs by firing action potentials at appropriate rates. Thus, when the level of GABRA increases it is reasonable to expect that the level of CLC will be lower in order to prevent electrical activity to become inhibited too much. And this is what a negative interaction from GABRA to CLC means. Another negative gene interaction relevant for the excitatory-inhibitory balance is the interaction between the gene for SCN and the NMDAR gene. Voltagegated sodium channels $(\mathrm{SCN})$ increase excitability of neurons, thus it would make sense when they are elevated in expression, that the expression level of the major excitatory receptor NMDAR is downregulated. Therefore the latter two predicted interactions for the normal EEG make sense from the neurobiological point of view. The other two negative interactions make less sense from the excitatory-inhibitory balance point of view. The analysis has shown that genes of both SCN and NMDAR have a negative influence upon the gene coding for potassium (kalium) channel $(\mathrm{KCN})$, thus leading to its downregulation when they are upregulated. $\mathrm{KCN}$ lets positive kalium ions out of the cell, thus making the cell interior more negative, and in such a way it counterbalances the action of excitation. Thus, it should be expected that upregulation of SCN and NMDAR leads to upregulation and not downregulation of $\mathrm{KCN}$ to counterbalance excitation.

In the set of Ws leading to the 2-3 Hz large-amplitude LFP resembling the electrical activity in the SWD in CAE EEG, we have found only one significant gene interaction. Our results show that there is a statistically significant negative interaction between the genes coding for GABRA and SCN. The negative interaction from GABRA towards SCN discovered by our analysis means, that actually excitatory sodium channels will be elevated in their levels, which enhances excitability of neurons, when GABRA levels are lowered. Our results do not predict that the genes for GABRA and/or SCN are mutated, but instead they predict that if GABRA is lowered in expression, this leads to an increased excitability of neurons via elevated expression of SCN channels, which is not a "healthy" gene interaction counterbalancing decreased neuronal inhibition. Our results show that even when none of the genes coding for excitatory and inhibitory receptors/ion channels is mutated, inappropriate interaction that violates the excitationinhibition balance can lead to an abnormal EEG. This prediction however, remains to be tested by genetic research.

\section{CONCLUSION}

In biological neural networks neuronal parameters that define the functioning of a neural network depend on genes and proteins in a complex way. Gene expression values change due to internal dynamics of the gene/protein regulatory network, initial conditions of the genes and external conditions. All this may affect gradually or quickly the functioning of the neural network as a whole. In our computer experiments, we have observed for example that different initial gene values can lead to the same outcome in terms of neuronal activity. Moreover, different types of gene interaction dynamics i.e. be it constant, periodic, quasiperiodic or even chaotic, can lead to a similar LFP of an associated SNN model, provided some crucial gene interactions are maintained. On the other hand, in the diseased brain, either altered initial conditions expressed in values of neural parameters, mutated genes and/or altered interactions within GRN lead to abnormalities in network activity. Realistic models of gene networks within neural networks should account for these processes.

In order to investigate these phenomena, we have set up a hierarchical model of EEG generation based on the idea of CNGM that is simple and biologically plausible. Although we have used a very simple model of spiking neuron, the SRM, the results can be generalized to more complex models since balancing excitation and inhibition is a general issue. Moreover, the model is robust with respect to model parameter values, which can be any values from the biologically realistic value ranges listed in Table II.

Presented case study on two kinds of EEG illustrates what kind of predictions can be obtained with the CNGM approach. In future, it may be worthy to identify the function and identity of other genes in the GRN to be able to draw predictions about their indirect influence upon neuronal dynamics. Furthermore, the GRN can be expanded to include genes for subunits of relevant proteins, thus enabling us to relax the one gene-one protein oversimplification, in which we treat groups of genes as one gene. And last but not least, finer analysis of the LFP/EEG signal by means of the wavelet transformation may be needed to lead to more precise quantitative comparisons.

Future research will concentrate around the following issues:

1) „What-if analysis“. What happens if one or few particular genes are erased or mutated? What happens if interactions within the GRN change due to some factors? What happens if external factors are included? In such a way our approach can serve as a noninvasive test.

2) Introduction of learning rule(s) into an ANN model and corresponding genes into the GRN.

3) Introduction of genes, which play role in synaptic connectivity and development of neural system.

4) Exploration of possibilities of modelling genetically caused brain disorders such as some epilepsies, Parkinson's disease, etc.

These goals may mean that instead of an SNN, another neural network model will be employed, since the general methodological principles of a CNGM introduced in this paper are of a general relevance. Our goal is to make useful 
predictions to aid the experimental testing.

Future analysis will be conducted when the same abstract GRN will be used for both states (normal and epileptic) and some genes/proteins will be altered thus simulating gene mutations, drug influences, etc.

\section{APPENDIX}

In this Appendix, we would like to show examples of SNN LFPs obtained in our computer simulations of CNGM.

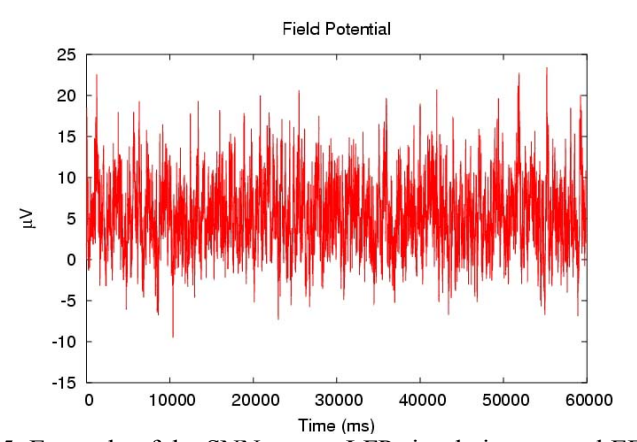

Fig. 5. Example of the SNN output LFP simulating normal EEG.

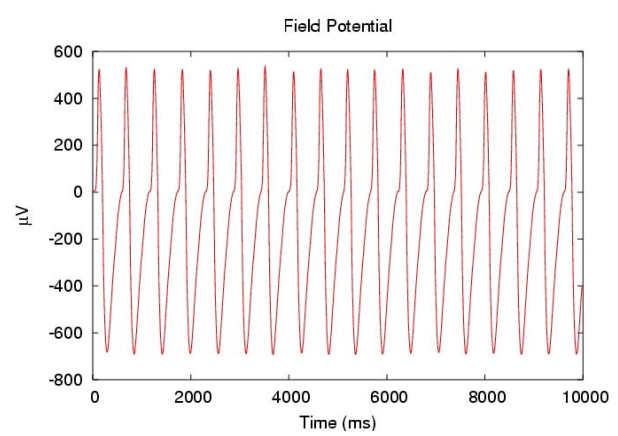

Fig. 6. Example of the SNN output LFP simulating seizure-like EEG.

\section{REFERENCES}

[1] C. E. M. van Beijsterveldt and G. C. M. van Baal, "Twin and family studies of the human electroencephalogram: a review and metaanalysis," Biological Psychology, 61, pp. 111-138, 2002.

[2] G. Buzsaki and A. Draguhn, "Neuronal oscillations in cortical networks," Science, 304, pp. 1926-1930, 2004.

[3] B. Porjesz, L. Almasy and H. J. Edenberg, "Linkage disequilibrium between the beta frequency of the human EEG and a $\mathrm{GABA}_{\mathrm{A}}$ receptor gene locus," Proc. Natl. Acad. Sci. USA, 99, pp. 3729-3733, 2002.

[4] N. Kasabov and L. Benuskova, "Computational neurogenetics," Journal of Computational and Theoretical Nanoscience, 1, 1, pp. 47$61,2004$.

[5] N. Kasabov, L. Benuskova and S. G. Wysoski, "A computational neurogenetic model of a spiking neuron," Proc. IEEE Intl. Joint Conference on Neural Networks, Montreal, Canada, 2005, vol. 1, pp. 446-451.

[6] D. C. Weawer, C. T. Workman and G. D. Stormo, "Modeling regulatory networks with weight matrices," Proc. Pacific Symp. Biocomputing, Hawai, 1999, vol. 4, pp. 112-123.

[7] L. F. A. Wessels, E. P. van Someren and M. J. T. Reinders, "A comparison of genetic network models," Proc. Pacific Symp. Biocomputing, vol. 6, Singapore, Hawai, 2001, vol. 6, pp. 508-519.

[8] P. D'Haeseleer, X. Wen, S. Fuhrman and R. Somogyi, "Linear modeling of mRNA expression levels during CNS development and injury," Proc. Pacific Symposium on Biocomputing, Singapore, 1999, vol. 4, pp. 41-52.
[9] N. Kasabov, Z. S. H. Chan, V. Jain, I. Sidorov and D. Dimitrov, "Gene regulatory network discovery from time-series gene expression data - a computational intelligence approach," in Lecture Notes in Computer Science, vol. 3316, N. R. Pal, N. Kasabov, R. K. Mudi, S. Pal and S. K. Parui, Springer-Verlag, Berlin, 2004,pp. 1344-1353.

[10] R. A. Deisz, "GABA $A_{B}$ receptor-mediated effects in human and rat neocortical neurones in vitro," Neuropharmacology, 38, pp. 1755$1766,1999$.

[11] A. Destexhe, "Spike-and-wave oscillations based on the properties of GABA $_{\mathrm{B}}$ receptors," J. Neurosci., 18, pp. 9099-9111, 1998.

[12] I. C. Kleppe and H. P. C. Robinson, "Determining the activation time course of synaptic AMPA receptors from openings of colocalized NMDA receptors," Biophys. J., 77, pp. 1418-1427, 1999.

[13] M. Simonato, R. Molteni, G. Bregola, A. Muzzolini, M. Piffaneli, L. Beani, G. Racagni and M. Riva, "Different patterns of induction of FGF-2, FGF-1 and BDNF mRNAs during kindling epileptogenesis in the rat," Eur. J. Neurosci., 10, pp. 955-963, 1998.

[14] Y. Li, J. Wang, J. G. Sheng, L. Liu, S. W. Barger, R. A. Jones, L. J. VanEldik, R. E. Mrak and W. S. Griffin, "S100 beta increases levels of beta-amyloid precursor protein and its encoding mRNA in rat neuronal cultures," J. Neurochemistry, 71, 4, pp. 1421-1428, 1998.

[15] C. M. Cheng, R. R. Reinhardt, W. H. Lee, G. Joncas, S. C. Patel and C. A. Bondy, "Insulin-like growth factor 1 regulates developing brain glucose metabolism," Proc. Natl. Acad. Sci. USA, 97, 18, pp. 10236$10241,2000$.

[16] M. Toth, J. Grimsby, G. Buszaki and G. P. Donovan, "Epileptic seizures caused by inactivation of a novel gene, jerky, related to centromere binding protein-B in transgenic mice," Nature Genetics, 11, 1, pp. 71-75, 1995.

[17] S. Zagulska-Szymczak, R. K. Filipkowski and L. Kaczmarek, "Kainate-induced genes in the hippocampus: lessons from expression patterns," Neurochemistry International, 38, pp. 485-501, 2001.

[18] K. Wojtal, A. Gniatkowska-Nowakowska and S. J. Czuczwar, "Is nitric oxide involved in the anticonvulsant action of antiepileptic drugs?," Polish J. Pharmacology, 55, pp. 535-542, 2003.

[19] W. Gerstner and W. M. Kistler, Spiking Neuron Models, Cambridge Univ. Press, Cambridge, MA, 2002.

[20] C. M. Colbert and E. Pan, "Ion channel properties underlying axonal action potential initiation in pyramidal neurons," Nature Neuroscience, 5, pp. $533-538,2002$.

[21] A. Erisir, D. Lau, B. Rudy and C. S. Leonard, "Function of specific $\mathrm{K}^{+}$ channels in sustained high-frequency firing of fast-spiking neocortical interneurons," J. Neurophysiol., 82, pp. 2476-2489,

[22] J. W. Freeman, Mass action in the nervous system, Academic Press, New York, 1975.

[23] R. Q. Quiroga, Quantitative Analysis of EEG Signals: Time-Frequency Methods and Chaos Theory., Institute of Physiology and Institute of Signal Processing. Medical University Lübeck: Lübeck, 1998.

[24] A. Destexhe, D. Contreras and M. Steriade, "Spatiotemporal analysis of local field potentials and unit discharges in cat cerebral cortex during natural wake and sleep states," J. Neuroscience, 19, 11, pp. 4595-4608, 1999.

[25] V. Crunelli and N. Leresche, "Childhood absence epilepsy: genes, channels, neurons and networks," Nature Rev. Neurosci., 3, 5, pp. 371$382,2002$.

[26] C. Marini, L. A. Harkin, R. H. Wallace, J. C. Mulley, I. E. Scheffer and S. F. Berkovic, "Childhood absence epilepsy and febrile seizures: a family with a $\mathrm{GABA}_{\mathrm{A}}$ receptor mutation," Brain, 126, pp. 230-240, 2003. 